\title{
Ouargla : du vieux port transsaharien à la métropole
}

\section{Ali Bensaad}

\section{(2) OpenEdition}

1 Journals

Édition électronique

URL : https://journals.openedition.org/encyclopedieberbere/2846

DOI : 10.4000/encyclopedieberbere.2846

ISSN : 2262-7197

Éditeur

Peeters Publishers

\section{Édition imprimée}

Date de publication : 2 juin 2013

Pagination : 5899-5911

ISBN : 978-2-7584-0194-0

ISSN : 1015-7344

\section{Référence électronique}

Ali Bensaad, «Ouargla : du vieux port transsaharien à la métropole », Encyclopédie berbère [En ligne], 36 | 2013, document O35, mis en ligne le 12 mars 2021, consulté le 17 février 2022. URL : http:// journals.openedition.org/encyclopedieberbere/2846; DOI : https://doi.org/10.4000/ encyclopedieberbere.2846

Ce document a été généré automatiquement le 17 février 2022.

(c) Tous droits réservés 


\title{
Ouargla : du vieux port transsaharien à la métropole
}

\author{
Ali Bensaad
}

1 Cité parmi les plus anciennes du Sahara, née comme l'essentiel des oasis du commerce transsaharien dont elle fut un important "port", Ouargla est aujourd'hui l'une des plus grandes villes sahariennes, métropole régionale affirmée, aux fonctions diversifiées. Regroupant une population d'environ 140.000 habitants et s'étendant sur $60 \mathrm{~km}^{2}$ dans le lit quaternaire de l'oued Mya, la ville est bordée d'une vaste palmeraie ou s'enfouit le vieux ksar alors qu'en lisière s'étale la ville nouvelle avec ses centres administratifs et militaires, ses zones industrielles et de services, liées surtout au pétrole, ses quartiers coloniaux, ses nouveaux lotissements d'habitat collectif et surtout individuel et l'inévitable étalement de l'habitat informel dont une part relève de la sédentarisation de nomades.

2 Si le Sahara est un espace où les villes naissent et meurent et que les villes mortes, prestigieuses ou anonymes, y sont légions, Ouargla, successeur comme capitale régionale de la prestigieuse Sedrata* (Isedraten) ensevelie depuis près d'un millénaire par les sables, est de ces autres villes historiques, tout aussi nombreuses, durablement enracinées au Sahara. Ouargla y a aussi renouvelé, dans un contexte contemporain radicalement transformé, les conditions d'affirmation de son poids et de son influence. Le commerce transsaharien, déclinant puis disparaissant, a privé les oasis sahariennes de leur ressource essentielle, les plongeant toutes dans la régression ; Ouargla, qui n'y a pas échappé, retrouvera plus tard un dynamisme nourri par les nouveaux enjeux sahariens : le contrôle d'un espace devenu stratégique, le renouveau agricole dans un contexte de crise de l'agriculture tellienne et, surtout, l'exploitation des ressources minières nouvellement accessibles, notamment la plus recherchée d'entre-elles, le pétrole.

3 La ville de Ouargla se situe dans le Bas-Sahara, la partie la plus active du Sahara algérien, qui regroupe $2 / 3$ des palmeraies et $2 / 3$ des habitants du territoire. Situé au nord-est du Sahara, le Bas-Sahara doit son appellation à sa topographie très basse, avec une altitude moyenne de $-40 \mathrm{~m}$ au-dessous du niveau de la mer, et non à sa latitude. Ce 
qui lui confère un climat particulier, avec de fortes températures et une grande siccité de l'air, propice à la maturation d'une variété de dattes, la Deglet-Nour, très réputée et ne poussant nulle part ailleurs, sauf bien sûr dans son prolongement tunisien. La particularité du Bas-Sahara s'explique par la disposition du bouclier saharien qui plonge à ce niveau vers le nord, donnant naissance à un vaste bassin sédimentaire en contrebas de l'Aurès-Nememcha*. S'y est constituée une couverture sédimentaire épaisse de 3 à 4.000 mètres, avec des couches aquifères et d'autres pétrolifères. Ces dernières constituent le gisement dit de Hassi-Messaoud du nom de la localité, centre de l'exploitation, située à $70 \mathrm{~km}$ de Ouargla, et dont les puits se distribuent en fait dans un rayon de $100 \mathrm{~km}$. Dans la foulée de l'exploitation pétrolière seront mises au jour les nappes aquifères profondes, considérables, dites du Continental intercalaire qui bouleverseront les données hydriques dans la région et viendront s'ajouter à celles déjà connues du Continental terminal.

4 La présence de ces ressources explique l'important investissement infrastructurel, économique et administratif dont bénéficiera cette région, son dynamisme et son poids, notamment démographique.

\section{Une histoire façonnée par le commerce transsaharien}

5 Mais même avant les bouleversements contemporains, et tout au long de l'histoire, cette région a été la plus habitée du Sahara. La disponibilité en eau n'y est pas étrangère. Les résurgences artésiennes et les techniques qu'elles permettaient, tels les forages spectaculaires de puits artésiens décrits avec force détails par Ibn Khaldoun (t. III, p. 299), permettaient déjà d'accéder aux nappes du Continental terminal qui serviront un développement hydro-agricole plus important et plus pérenne que dans le reste du Sahara. Mais c'est par l'association de cet artésianisme avec leur fonction de relais sur les routes transsahariennes que s'explique la relative opulence de ces oasis. En effet, si l'eau a donné une viabilité à ces oasis, elle n'en justifie pas l'existence. Celles-ci sont nées pour la plupart de façon volontariste, comme relais pour soutenir ces échanges dans un milieu aride et hostile. Elles ont vu le jour le plus souvent entre le $\mathrm{VII}^{\mathrm{e}}$ et le $\mathrm{XVIII}^{\mathrm{e}}$ siècle, deux dates marquant respectivement l'apparition du commerce transsaharien et le début de son déclin. Plus relais dans un système relationnel qu'agrosystème, elles dépendaient fortement de ce commerce. La preuve en est qu'avec le déclin de celui-ci, elles deviendront des poches de misère comme les redécouvriront explorateurs européens et militaires français au XIX siècle. L'agriculture en tant que telle, plus exigeante en travail et en eau sous le climat saharien, n'avait de rentabilité que « perfusée » par le commerce transsaharien qu'elle soutenait. Ce commerce, source principale de richesses, était aussi source de conflits : pour le maîtriser, on tendra à en contrôler d'abord les axes et donc les villes étapes dont la place et l'évolution sont souvent tributaires de ces conflits. L'histoire de Ouargla et son devenir illustrent la force de ce lien.

Deux dates et deux événements clés dans l'histoire de ce commerce marqueront la destinée de cette ville qui y était déjà fortement liée de part sa composant humaine berbère ibadite. Convertis au mouvement de dissidence religieuse, le kharijisme dans sa version ibadite, les Berbères du Maghreb, grâce à leur vaste réseau relationnel et de solidarités, notamment les points d'ancrage que constituaient les agglomérations contrôlées par des foyers ibadites (Sigilmassa*, Tahert*, le Fezzan, l'île de Djerba*, les 
villes du Mzab*, le pays de Ouargla, Ghadamès* ou le Djebel Nefoussa*), allaient asseoir leur suprématie commerciale. Ouargla était déjà insérée dans ce réseau bien avant que la chute en 909 de Tahert, centre du pouvoir ibadite Rustumide, ordonnateur du commerce transsaharien, n'entraîne un redéploiement territorial ibadite dans la vallée de Ouargla et y transfère les compétences, les moyens et le pouvoir liés à ce commerce. Même si le pôle en était alors Sedrata, à $15 \mathrm{~km}$ au sud de Ouargla, cette dernière profitera du rayonnement de la première et lorsque celle-ci sera détruite en 1229, elle en prendra le relais comme capitale régionale et comme centre d'un commerce transsaharien alors florissant.

\section{Ouargla, port majeur du commerce transsaharien et capitale régionale}

7 La fondation de Ouargla remonte au tout début du VIII siècle. Composée d'abord de quelques bourgades voisines les unes des autres, celles-ci finirent par se réunir et former une ville déjà importante pour l'époque. On retrouve chez tous les géographes et voyageurs arabes, malgré des imprécisions sur le lieu, l'idée d'un processus d'agglomération de ksars, avec parfois même des précisions communes à tous, comme celle évoquant, à l'origine, sept forteresses berbères, que l'on retrouve aussi bien chez le géographe El-Bekri, le voyageur et géographe Yāqūt al-Ḥamawīi ou dans le manuscrit anonyme du Kitāb al-istibșār. Le géographe Ibn Sa'īd al-Maghrībīi ${ }^{2}$, décrivant Ouargla cinq siècles plus tard, évoque encore la visibilité de forteresses agglomérées. Ce processus est confirmé par Ibn Khaldoun qui écrit (t. III, p. 286) que les Ban̄u Wārklā ont fondé Ouargla alors qu'ils ne formaient encore qu'un petit groupe ; ce furent d'abord quelques châteaux forts rapprochés, puis le nombre des habitants de ces châteaux augmenta, ils nouèrent de bonnes relations et une ville se développa. L'appellation Ban $\bar{u}$ Wārklā est utilisée tout près de la fondation de la ville, à la fin du $\mathrm{IX}^{\mathrm{e}}$ siècle, par le polygraphe Ibn Qutayba ${ }^{3}$ qui parle de fondation et de développement de la ville par les «Banū Wārklā, peuple zénatien descendant du Ferini, fils de Djana [...] de toutes ces tribus celle de Wārklā est maintenant la plus connue». Mais il semble bien que l'appellation Banū Wārklā recouvre une agglomération de clans berbères notamment les Beni Brahim, les Beni Sissine et les Beni Ouaguine qui continuent d'ailleurs à donner leur nom aux trois quartiers de l'actuel ksar dont les habitants, parlant une variété de berbère, la Teggargrent (cf. notice 036), sont toujours désigné comme "Ouarglis", nettement distingués des nomades sédentarisés et autres populations qui arriveront à la fin de la période coloniale.

8 L'importance de Ouargla dans le commerce transsaharien et dans le réseau ibadite est anciennement avérée, bien avant la création et la chute de Sedrata. La conversion de la région à l'ibadisme est effective dès le VIII ${ }^{\mathrm{e}}$ siècle et la route entre Tahert et Ouargla est très fréquentée dès la fondation de l'imamat rustumide. Abou Zakaria, dans son Kitāb as-sìra mentionne ainsi, par exemple, la ville comme étape entre le Djebel Nafoussa et Tahert. La chute de cette dernière en 909, en entraînant une migration des Ibadites vers l'oasis de Ouargla, la reconstitution de leurs réseaux commerçants et la fondation, à $15 \mathrm{~km}$ au Sud de Ouargla, de Sedrata (Isedraten) - probablement sur un établissement berbère antérieur - qui deviendra un pôle majeur de ce commerce, fera du pays de Ouargla un lieu de prospérité. Le Kitāb al-istibșār décrit ainsi le balad Warğalān comme une région fertile, abondant en palmiers et en jardins, qui produit beaucoup de 
céréales, compte de nombreux bestiaux et est riche en eau. Bien que Sedrata en était la capitale, Ouargla est souvent décrite comme une cité prospère même si la confusion a pu régner chez les auteurs arabes qui appliquaient le nom de Ouargla à l'ensemble des localités de la région, sans distinguer Sedrata de Ouargla. Une confusion qui s'expliquerait par la dispersion et l'abondance dans la région de Ouargla de hameaux fortifiés (325 villages et 1.051 fontaines selon un manuscrit ancien) et les nombreux noms, variant selon les dialectes, qui leur sont attribués. C'est ainsi que pour le géographe El-Bekri, dans son Kitāb al-Masālik wa al-Mamālik, ou pour l'auteur anonyme du Kitāb al-istibșār ou pour le voyageur et géographe Yāqūt al-Hamawī, dans son Mu'ğam al-buldān, il n'est jamais question de Sedrata mais seulement de Ouargla. Ce n'est qu'à la seconde moitié du XIII ${ }^{e}$ siècle que la description fournie par le géographe Ibn Sa'îd est clairement celle de Ouargla : il évoque une contrée (bilād) riche en palmiers dont la ville principale s'appelle Wārkalān. Entre temps était intervenue la destruction de Sedrata, en 1229 (après une première destruction partielle en 1075) et Ouargla émerge comme capitale et centre du commerce transsaharien. Ainsi, à la fin du XIV siècle Ibn Khaldoun écrit : « De nos jours, la ville de Ouargla est la porte du désert par laquelle les voyageurs qui viennent du Zab doivent passer quand ils veulent se rendre au Soudan avec leurs marchandises » (t. III, p. 286).

9 La ville, longtemps gouvernée par des chefs prenant le titre de sultan, est juchée sur une butte et défendue par une enceinte ; un fossé et des sebkhas la ceinturant. Un point d'eau et la tombe de Sidi El-Ouargli auraient été les points initiaux de la structuration de l'habitat organisé en trois quartiers ethniques précédemment cités. Deux mosquées, l'une ibadite, l'autre malékite, sont en position centrale et témoignent de l'histoire religieuse de la ville et notamment de l'emprise malékite qui a succédé à l'ibadite. Mais l'élément véritablement structurant de la ville, ce sont les trois pistes sur lesquelles ouvrent chacune des trois portes de l'enceinte et menant respectivement sur N'goussa* vers le nord, Ghardaïa vers le nord-ouest et El-Goléa vers le sud, sur la route du "Soudan". Ce sont ces trois pistes qui ont structuré un tracé de la ville centré sur le vieux marché, l'élément fonctionnel central attestant de la primauté de la fonction marchande.

\section{Marginalisation sur les routes commerciales, régression et latence}

Ouargla restera pendant quelques siècles une ville prospère et centrale sur les routes transsaharienne jusqu'à ce qu'au XVII ${ }^{e}$ siècle, la concurrence des nouvelles routes océanes finisse par ruiner le commerce transsaharien et fasse tomber Ouargla, comme les autres villes sahariennes, dans la stagnation puis la régression. Il n'y a pratiquement plus d'informations sur la ville, qui ne suscite plus l'intérêt des chroniqueurs ; mais on peut mesurer le degré de régression par le fait qu'au premier recensement réalisé par les Français en 1882, la population y était à peine de 2.000 habitants alors qu'elle avait pu atteindre plusieurs dizaines de milliers de personnes dont, il est vrai, une partie flottante.

11 La colonisation française $e^{4}$ 'atteint Ouargla qu'en 1852 alors que la ville abritait Mohamed Ben Abdellah, originaire de Tlemcen qui, après plusieurs pérégrinations en Algérie et en Orient, s'y fixa pour continuer la résistance contre l'armée française et s'y fit nommer Cherif de la ville. Elle mettra deux décennies avant de s'y affirmer, avec des 
flux et des reflux, notamment en raison de la résistance rencontrée dans la région et au gré des fluctuations des alliances et allégeances tribales.

La première empreinte coloniale sur la ville sera, comme acte de rétorsion lié à cette résistance, une percée réalisée dans le ksar donnant naissance à la "rue de Rivoli" ou "rue des cents colonnes" qui ouvre l'accès au cœur de la ville, le marché, pour lui assurer une défense plus aisée. Cette percée sera réalisée aux dépends des habitations des Beni Sassine qui avaient soutenu la révolte de Bouchoucha en 1864.

13 Jusqu'à la veille du centenaire de la colonisation, l'empreinte de celle-ci restera surtout militaire ; en plus des percées dans le ksar et du remblaiement du fossé périphérique transformé en boulevard, elle consistera en la construction de bordjs (forts). Il en sera réalisé trois : le Bordj Chandez, le Bordj Luthaud et le Bordj du Génie. Ils deviendront par la suite les éléments structurants du développement de la ville.

14 Il faudra attendre les années trente, et plus précisément 1927, avec l'arrivée du colonel Carbillet pour qu'il y ait un développement de la ville. Il conçut, sur un terrain vierge, une ville nouvelle juxtaposée au ksar, avec des voies parallèles et perpendiculaires traçant un réseau en damier bordé de jardins. Bien qu'elle s'étendait sur le double de la surface du ksar, le terme de ville doit être relativisé. En effet, peu peuplée, elle abritait surtout des équipements et des logements destinés aux militaires et fonctionnaires français. Le long de l'allée du Général Laperrine, reliant le Bordj Luthaud au ksar, d'autres équipements typiquement coloniaux seront réalisés : l'église, un musée et des hôtels ; une orientation Nord-Ouest/Sud-Est est ainsi imprimée au reste des rues. Alors que le ksar est resté centre social et commercial pour les populations autochtones, la ville nouvelle s'impose comme centre fonctionnel et administratif.

15 Après 1940, la ville continue de s'étendre selon les plans du colonel Carbillet et surtout à se densifier avec, par exemple, la construction de la cité Sélice destinée à accueillir les civils français. Parallèlement, s'enclenche un processus de sédentarisation de familles nomades implantées par regroupements tribaux sur des terres arch, formant ce qui a été appelé «quartiers de nomades sédentarisés »: Beni Thour, M’khadma et les Saïd Otba, du nom des tribus d'appartenance des nomades installés. La densification de ces quartiers s'effectue en même temps que leur transformation en dur.

16 À la fin de la période coloniale, vers 1960, Ouargla était devenue un important centre administratif et militaire ; les débuts de l'exploitation du pétrole et du développement des infrastructures avaient attiré une nouvelle main-d'œuvre et de nombreux nomades s'y étaient sédentarisés avant même la découverte du pétrole ; 1.200 Français s'y étaient implantés avec ce que cela suppose comme besoins en main-d'œuvre autochtone. Malgré cela, la population totale, hors Français, n'y était que de 6.600 habitants. Démographiquement, Ouargla était encore si peu une ville.

\section{Un devenir de métropole porté par le volontarisme étatique}

17 C'est avec l'indépendance que Ouargla retrouve et développe tous les attributs d'une métropole. Plus que le pétrole qui en a fourni les moyens, les enjeux géopolitiques internes et externes liés au contrôle des espaces sahariens ont été le facteur principal de promotion au travers d'une politique volontariste de contrôle territorial. Celle-ci va se concrétiser symboliquement par la tenue en 1966 à Ouargla du premier conseil des 
ministres hors capitale pour marquer le lancement du «Plan spécial Oasis». Ce plan fut initié dans un contexte où les frontières sahariennes étaient partout contestées, entraînant même un conflit algéro-marocain ${ }^{5}$. Le Bas-Sahara et son bassin sédimentaire, avec ses gisements pétroliers et aquifères s'étendant à la Tunisie et à la Libye, était également objet de contestation pour son partage alors qu'il recelait les plus importantes ressources pétrolières de l'Algérie et regroupait la plus grande partie de sa population saharienne. Mais l'enjeu du contrôle des oasis du Bas-Sahara se doublait d'un enjeu interne : la région fut en effet le lieu d'une contestation interne au pouvoir menée en 1964 par le colonel Chaabani ${ }^{6}$.

18 Le volontarisme géopolitique du régime trouvera dans le pétrole un puissant moyen de son action, tout en sécurisant celui-ci comme ressource. Son exploitation aura contribué au développement de Ouargla à travers la construction de routes, d'un aérodrome et de nombreux bâtiments mais aussi en générant une masse salariale considérable qui a stimulé le développement d'un important secteur commercial. Même si elle n'est pas située au cœur du bassin pétrolifère, Ouargla fait fonction de grand centre urbain servant de base logistique et de base arrière pour les pétroliers. Par ailleurs les récentes découvertes de nouveaux champs pétrolifères à l'ouest de Ouargla renforcent sa centralité.

Mais c'est la promotion administrative, portée à bout de bras par l'État, qui fera véritablement décoller Ouargla et la portera au rang de métropole, en multipliant ses attributs et ses activités, en les diversifiant et en accroissant spectaculairement sa population. La promotion de Ouargla s'est faite à un double niveau. En même temps qu'elle devenait siège de wilaya (préfecture), avec tous les équipements, activités et services relevant de ce niveau de gestion, elle devenait aussi capitale administrative d'une très vaste région, la wilaya des Oasis, couvrant tout le nord-est du Sahara. La masse salariale qui irrigue ainsi la ville stimule le développement du commerce et des services et, aux immigrants du Nord venus pour les activités pétrolières ou affectés pour étoffer l'appareil administratif, s'ajoutent des commerçants et prestataires de service attirés par le dynamisme de la ville; beaucoup sont Kabyles, par exemple dans la restauration qui fonctionne sur le mode de la « filière ethnique ». Entre 1960 et 1975, le nombre de commerces a été multiplié par 6 et la tendance s'est maintenue jusqu'aux années 1990.

20 Mais c'est bien sûr le rayonnement administratif et économique à l'échelle saharienne qui attire et brasse les populations de la région. Malgré le nouveau découpage administratif de 1974 qui réduisait son territoire en le scindant en six nouvelles wilayas, Ouargla a gardé une influence de dimension régionale notamment parce que les pouvoirs publics lui ont dévolu des rôles à échelle régionale et y ont implanté des institutions de développement, financières, de formation ou éducatives à vocation saharienne. Elles viennent s'ajouter au rôle de siège du commandement de la quatrième région militaire qui en fait un lieu de nombreuses garnisons et un avant-poste militaire stratégique pour l'Algérie dans l'ensemble saharien. C'est aussi aujourd'hui à une dimension transnationale saharo-sahélienne que s'ouvre Ouargla. Carrefour d'échanges important et obligé sur le grand axe méridien qui, depuis les villes portuaires de Skikda et Annaba dessert le Sahara, Ouargla se réinsère également dans le réseau des échanges transsahariens revivifiés. Ceux-ci s'incarnent notamment dans l'apparition, dans la ville, de migrants subsahariens transitant vers le nord et/ou travaillant dans les grandes exploitations agricoles qui se sont multipliées ces dernières années. On les 
retrouve souvent dans les parties dégradées du ksar où ils côtoient, sans mélange, les vieux habitants hartanis*, juxtaposant ainsi deux phases de l'expression de l'africanité de ce ksar.

\section{Diversification fonctionnelle et complexification urbaine d'une métropole affirmée}

21 Promotion administrative, dévolution d'un rôle structurant et de commandement à l'échelle régionale, fonction de carrefour entre le nord du pays et sa profondeur saharienne, et enfin centralité dans l'exploitation d'une ressource cruciale, le pétrole, autant d'atouts qui se sont additionnés pour donner à la ville une complexité sans égale dans le Sahara algérien et un statut de métropole, qui se traduit évidemment dans les effectifs de sa population.

Ainsi depuis le recensement de 1966, Ouargla double presque en moyenne sa population tous les dix ans. Celle-ci passe de 20.000 à 47.000 en 1977, à 82.000 en 1987 puis à 140.000 habitants en 1998. Alors qu'elle comptait à peine la moitié de la population de Touggourt avant l'indépendance, elle a aujourd'hui supplanté celle-ci et Ghardaïa, s'imposant en véritable capitale saharienne. Ces dernières années, les effectifs se sont quelque peu tassés (135.000 habitants au recensement de 2008) malgré un mouvement d'immigration observé pendant la "décennie noire " (1990-2000) où, réputée sûre puisque située dans le périmètre le plus sécurisé d'Algérie car assurant les rentrées en devises du pays, Ouargla a accueilli des familles du nord fuyant la violence. Le mouvement a pu n'être que temporaire avec le retour de la sécurité dans les régions d'origine, mais entre-temps cela a donné naissance à des quartiers spontanés comme celui de Sokra.

Cette stabilisation de population marque la maturation d'une ville qui ne croît plus par l'apport de l'exode mais dont la croissance naturelle épouse aussi la baisse tendancielle générale de la natalité. Elle marque aussi les limites du modèle de croissance de la ville et de ses deux principaux facteurs : l'essoufflement du volontarisme d'Etat notamment avec le contrechoc pétrolier des années 1980 et 1990 et les réajustements structurels qui ont amputé entreprises et services publics, et l'extrême libéralisation du secteur pétrolier qui s'est ainsi exonéré d'une "fonction sociale " que lui faisait supporter l'État. Cela se traduit par une forte tension sociale qui secoue la ville depuis l'année 2008, avec des pointes comme les émeutes de juin 2010 ou celles d'octobre 2011.

Néanmoins cette croissance a transfiguré le paysage urbain de Ouargla au point qu'après une intense densification, l'urbanisation a enjambé l'obstacle du lac et des sebkhas et, dans une course aux terrains, a atteint les vieux ksour villageois de Rouissat au sud, Chott, Sidi Khouiled et Adjaja à l'est et Boumendil à l'ouest. Le Ksar, bien que l'un des mieux conservés du BasSahara et que les trois quarts de ses habitants soient des natifs auxquels s'ajoutent d'autres Ouarglis ancrés dans la culture citadine, atteint quand même les limites de la saturation. En 1977, par rapport au premier recensement de l'époque coloniale (1882), ses effectifs ont été multipliés par cinq (10.400 habitants). Malgré l'actuel reflux (8.000 habitants au milieu des années 2000 selon une enquête de terrain), il connaît une grande densification qui, pour contourner l'interdiction de construction en hauteur, s'est exprimée dans la construction de passages couverts (les rues sont couvertes de pièces d'habitation) ou le grignotage des jardins intérieurs. 
Néanmoins le ksar reste le référent culturel et spirituel et continue à jouer le rôle de centre de la ville, quand bien même les autres quartiers ont pu s'imposer par des spécialisations fonctionnelles (administratives, sanitaire, éducatives, etc.).

La densification des «quartiers nomades " aux abords du ksar se poursuit aussi, avec aujourd'hui une deuxième et parfois une troisième génération de constructions dans la continuité de la "maison mère». Aussi la densité y atteint 110 à 150 habitants à l'hectare quand elle n'est en moyenne que de 80 dans la ville en raison de la prédominance de l'habitat individuel. Cependant les besoins importants ont poussé les pouvoirs publics à lancer des programmes de logements collectifs et les grands ensembles aux noms impersonnels de "cité des 500 logements", "cité des 150 logements", surgissent aux franges de la ville, faisant passer la part du logement individuel de $80 \%$ dans les années 1980 à $60 \%$ aujourd'hui.

Cette complexité urbaine a eu pour effet de déplacer le centre de gravité de la ville, longtemps situé à la sortie du ksar et au contact du quartier colonial, pour le transporter au point de rencontre de quatre quartiers différents, expression de la nouvelle complexité de la ville: le quartier de Beni Thour (anciens nomades), le quartier administratif à proximité du siège de la wilaya, le «triangle vert» des militaires (quartier cossu et verdoyant de la nomenklatura militaire, hérité de l'armée coloniale), le quartier Sidi Boughafla abritant les immigrés originaires du nord (cadres pétroliers et administratifs). C'est ici le lieu de négoce d'une ville qui attire dorénavant, notamment pour son marché hebdomadaire, des commerçants venant de tout l'Est algérien et de l'Algérois.

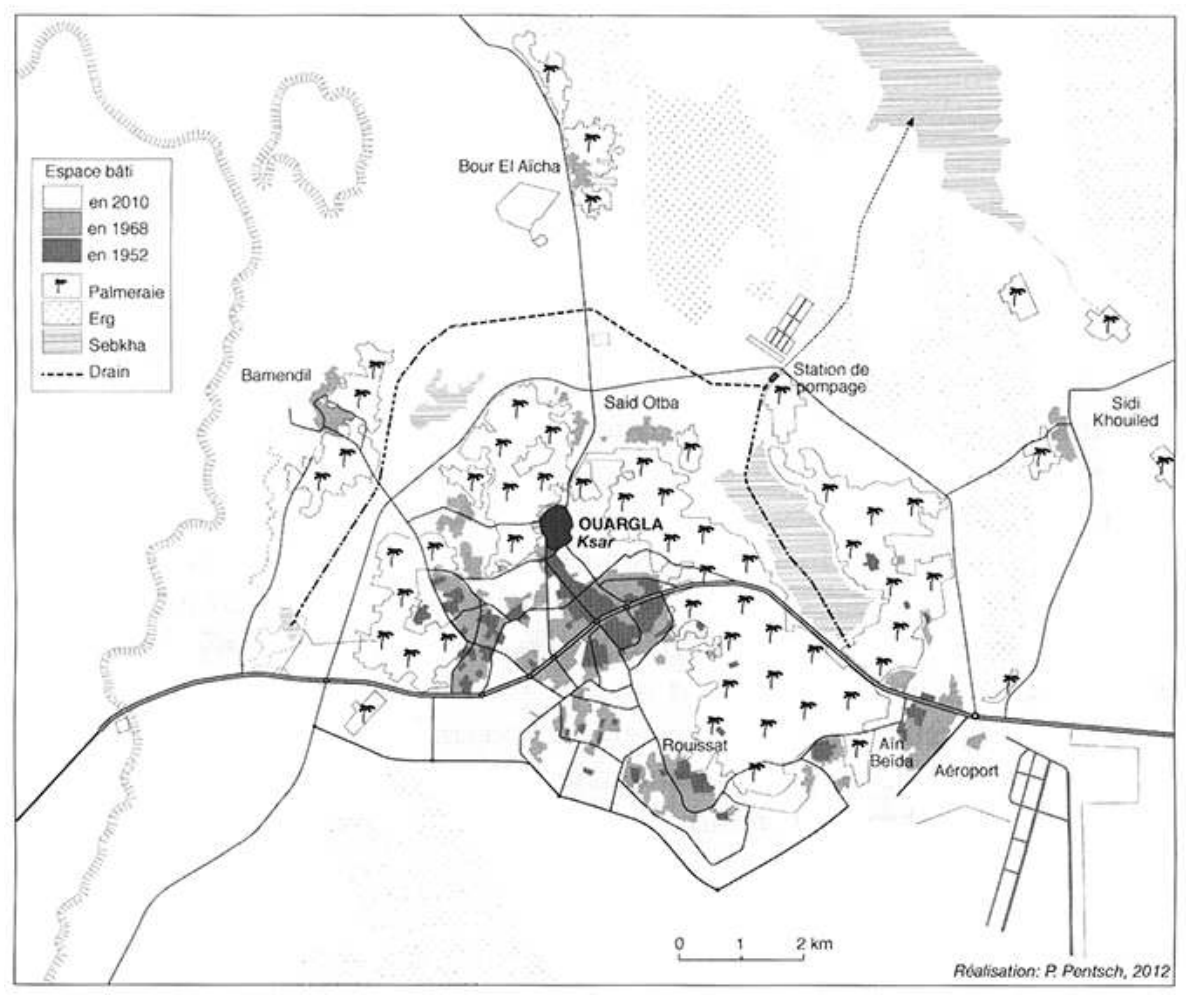

Fig. 1 : eVOLUtion de L'EMPRISE dE LA VILLE DE OUARgLA : 1952, 1968, 2010. 


\section{Une durabilité menacée et un devenir en question}

27 Cette urbanisation effrénée et longtemps euphorique bute désormais sur des limites qui peuvent même remettre en cause sa durabilité. Ouargla est maintenant une ville " assiégée » par ses eaux usées et par les eaux d'irrigation de palmeraies démultipliées, des eaux asphyxiant les cultures et les palmiers, mais menaçant aussi habitations et bâtiments publics. L'accès aux eaux profondes du Terminal intercalaire, situées à 1500-2000 $\mathrm{m}$ de profondeur, a mis à la disposition de l'urbanisation et de l'agriculture des quantités considérables d'eau. Les dotations théoriques en eau dans les villes du Bas-Sahara surclassent de loin celles des villes du nord et se situent au niveau des villes européennes : 300 à $500 \mathrm{~L} /$ habitant/jour, même si dans la pratique les déperditions en gaspillent la majeure partie. Mais ces eaux ont aussi permis un développement exceptionnel de l'agriculture. L'Etat, grâce à ces eaux et à l'absence d'obstacle foncier, a cherché à compenser au Sahara les déboires de l'agriculture tellienne, encourageant entrepreneurs agricoles locaux et du nord. Cependant l'environnement habitué à des débits d'eau de 1 litre/seconde provenant des puits de la nappe phréatique superficielle et 40 litres/seconde pour ceux de la nappe du Continental terminal, se retrouve avec des débits de l'ordre de $250 \mathrm{l} / \mathrm{s}$ ! Et les eaux continuent toujours à être évacuées, comme précédemment, dans la nappe phréatique superficielle. Tant qu'elles n'étaient pas importantes, elles y étaient recyclées naturellement; or, les nouveaux débits, considérables, dépassent les capacités de cette nappe qui gonfle, suscitant une remontée des eaux.

La sebkha entourant Ouargla, noyée par la remontée des eaux urbaines et d'irrigation, suggère cette image de siège. Le drain de 3 à $4 \mathrm{~km}$ qui aboutissait à la sebkha s'est trouvé à son tour saturé au point de refouler l'eau vers la palmeraie puis vers la ville, obstruant et endommageant des canalisations d'assainissement déjà vétustes et sources de fuites. Les palmiers étouffent et le bâti connaît des dégâts. Ceux-ci sont paradoxalement plus importants là où, après imbibition du sol, un assainissement et un assèchement ont été entrepris, tassant le sol et déstabilisant le bâti. La maison d'arrêt illustre de manière spectaculaire ce nouveau danger: non seulement le bâtiment est endommagé, mais ses fondations ne sont plus au même niveau.

A la crise sociale et à l'essoufflement de son modèle de croissance, Ouargla ajoute donc une crise environnementale aiguë. Elle est à un autre tournant capital de son devenir qui résume bien celui du Sahara et de son développement.

\section{BIBLIOGRAPHIE}

ABOU ZAKARIA (Yahya ibn Abī Bakr al-Warjālanī), Kitâb As-sīra... Chronique d'Abou Zakaria, traduite et commentée par Emile Masqueray, Alger, A. Jourdan, 1978, 410 p. (Il existe plusieurs rééditions en fac-similé).

BENSAAD A. (Dir.), 2011 - L'eau et ses enjeux au Sahara, Paris, Karthala, 252 p. 
BENSAAD A., 2006 - «Le paradoxe environnemental des villes sahariennes », in Elisabeth DorierApprill, Ville et environnement, SEDES/Armand Colin, Paris, 2006, p 435-453.

BISSON J., 2003 - Mythes et réalités d'un désert convoités. Le Sahara, Paris, L'Harmattan, 480 p.

BISSON J., 1983 - « Les villes sahariennes : politique volontariste et particularisme régionaux », Maghreb-Machrek, 100, Paris, La Documentation française, p. 25-41

BLANCHET P., 1900 - «L'oasis et le pays de Ouargla », Annales de Géographie, 1900, t. 9, nº 44.

p. $141-158$.

CôTE M, (Dir.), 2005 - La ville et le désert. Le Bas-Sahara algérien, Paris, Karthala, 312 p.

CÔTE M, (Dir.), 2002 - Le Sahara, cette « autre Méditerranée », Méditerranée, 3-4, 2002.

EL-BEKRI, Kitāb al-masālik wa-l-mamālik - Description de l'Afrique septentrionale, éditeur-trad. W. Mac Guckin de Slane, Paris, Adrien-Maisonneuve, 1965.

IBN KHALDOUN, Histoire des berbères et des dynasties musulmanes de l'Afrique septentrionale, t. III, éditeur-trad. W. Mac Guckin de Slane, Paris, Paul Geuthner, 1978 ; rééd. Alger, BERTI Editions, 2003.

IBN QUTAYBA ibn Muslim, Kitāb al-‘Arab, Farīd ‘Abd al-‘Azīz Al-Ğundī (éd.), Beyrouth, 1990. IBN SA‘ĪD AL-MAGHRĪBĪ, Kitāb al-ğugrāfiyā, Ismā‘īl Al-‘Arabī (éd.), Beyrouth, 1970.

Kitāb al-istibșār (Anonyme), traduction E. Fagnan, in : Recueil de la Société Archéologique de Constantine, 1898.

PREVOST V., 2008 - « Une tentative d'histoire de la ville ibadite de Sadrāta », Mélanges de la Casa de Velázquez, 38-2, p. 129-142.

ROUVILLOIS-BRIGOL M., 1975 - Le pays de Ouargla (Sahara alghérien). Variations et organisation d'un espace rural en milieu désertique, Paris, Sorbonne (Département de Géographie), 390 p.

YĀQŪT AL-ḤAMAWİ, [Šihāb al-Dīn Abū 'Abd Allāh], Mự̆am al-buldān (vol.5/7), Farīd 'Abd al-'Azīz AlĞundī (éd.), Beyrouth, 1990.

\section{NOTES}

1. Yāqūt ibn 'Abdullah al-Rūmī al-Ḥamawī, biographe et géographe : 1179-1229.

2. 'Ali ibn Mūsa ibn Sa'îd al-Maghrībī, connu aussi sous le nom de Ibn Sa'īd al-Andalūsī, géographe, historien et poète andalou : 1213 (Grenade) - 1286 (Tunis).

3. Polygraphe sunnite d'origine persane, Abū Muhammad 'Abd Allah ibn Muslim al-Dīnawārī ibn Qutayba est né à Koufa (828) et mort à Baghdad (889).

4. Ouargla devint poste avancé et commandement militaire français pour l'ensemble du Sahara algérien; la ville fut de ce fait, à maintes reprises, un poste d'observation et d'étude des populations berbères sahariennes et de leur langue : la plupart des berbérisants pionniers du $\mathrm{XIX}^{\mathrm{e}}$ siècle y ont séjourné, notamment pour entrer en contact avec des Touaregs, caravaniers ou prisonniers de guerre, qui furent leurs premiers informateurs (Hanoteau, 1860; Cid Kaoui, 1883...). NDLR.

5. Le conflit algéro-marocain sur la frontière ouest du Sahara débute en octobre 1963 et donne lieu à de violents affrontements militaires (dits « Guerre des sables ») ; un cessez-le-feu est signé le 5 novembre 1963. Ce sera le début d'une longue et durable relation de tension entre l'Algérie et le Maroc, dont le dossier du Sahara occidental ne sera que le dernier avatar. Des tensions existent 
aussi avec la Tunisie, avec des incidents sporadiques moins graves, autour de la zone frontalière (pétrolifère) d'El-Borma (Bir Romane - Garetel-Hamel); le bornage de la frontière ne sera définitif qu'avec le traité algéro-tunisien de janvier 1970 (fixant le tracé de la frontière entre Bir Romane et la frontière libyenne). Au sud, la première révolte des Touaregs de l'Adghagh des Ifoghas contre le pouvoir malien commence en mai 1963 et montre la porosité de la frontière : le territoire algérien sert de base de repli aux révoltés; les chefs, qui s'y réfugient après la défaite militaire, seront livrés par Alger à Bamako et seront exécutés. Dans les années qui suivront l'indépendance, une réelle tension existe donc sur toutes les frontières sahariennes de l'Algérie. NDLR.

6. Le colonel Mohamed Chaabani (né en 1934 à Oumache, Biskra) est responsable de la Wilaya VI (Sahara) pendant la guerre de libération algérienne, à partir de 1959. Après l'indépendance, il commande la $4^{\mathrm{e}}$ région militaire (oasis du Bas-Sahara). Après la prise de pouvoir par Ahmed Ben Bella (et son allié militaire Boumediene), le colonel Chaabani prend position contre le régime qu'il juge autoritaire. En 1964, il participe à une révolte des wilayas. Ben Bella accuse Chaabani de complot contre le FLN et l'État et de tentative de sécession du sud algérien et d'accaparement de son pétrole - la presse officielle de l'époque se déchaîne contre celui qui « voudrait se tailler un fief dans le Sud »... Le colonel Chaabani est arrêté le 8 juillet 1964 ; il est jugé le 2 septembre 1964 par une cour martiale, condamné à mort et exécuté le 3 septembre 1964. Il sera réhabilité le 24 octobre 1984 par décret présidentiel. NDLR.

\section{INDEX}

Mots-clés : Géographie, Sahara, Ville 